\title{
On the Observed Momentum Flux by Standing Eddies
}

\author{
Manktn MaK \\ Laboratory for Atmospheric Research, University of Illinois, Urbana 61801
}

29 August 1977 and 1 November 1977

\begin{abstract}
An analysis of four years of $500 \mathrm{mb}$ height data unexpectedly reveals that the standing waves which are of planetary scale meridionally transport an amount of momentum comparable to that of transient waves. Evidence is presented to show that the statistics concerning the standing waves are difficult to determine accurately. These statistics are particularly sensitive to the errors which arise from the large gaps in the longitudinal distribution of existing radiosonde stations. Planetary waves may play a more important role in the troposphere than past investigations might tend to suggest.
\end{abstract}

\section{Introduction}

- This note reports an unexpected find on the meridional flux of momentum by standing eddies at $500 \mathrm{mb}$. Past observational studies appeared to have led to a general consensus that transient eddies transport much more momentum poleward than standing eddies do. For instance, Lorenz $(1967$, p. 81$)$, upon reviewing the atmospheric statistics, stated that in the Northern Hemisphere the transport of angular momentum by standing eddies accounts for about $20 \%$ of the total eddy transport. In the course of working on another (C) 1978 American Meteorological Society 
problem with the use of four recent years of daily $500 \mathrm{mb}$ height data, I computed these statistics using geostrophically determined wind. The result for the standing-eddy flux turns out to be about $75 \%$ of the transient-eddy flux over those latitudes where both are important. Within the winter season, the former even slightly exceeds the latter. It follows that a substantial portion of the annual averaged transient-eddy flux is attributable to those long-period waves.

More recent observational works on atmospheric statistics have made no comment on the basic statistics such as the one under consideration. Instead, they put the emphasis on the spatial and/or temporal spectral characteristics of various variances and covariances (e.g., Kao and Kuczek, 1973; Blackmon, 1976; Pratt and Wallace, 1976; Blackmon et al., 1977; Pratt, 1977). The most comprehensive investigations of atmospheric statistics to date are perhaps those by Oort and Rasmusson (1971) and Newell et al. (1972), to be referred as OR and NKVB, respectively. A close intercomparison among the results of $\mathrm{OR}, \mathrm{NKVB}$ and mine reveal that the three sets of estimates for the standing-eddy momentum flux significantly differ from one another. It is therefore worth calling attention to this uncertain state of affairs.

\section{Data and results}

The data used in this study consist of an uninterrupted four-year record of daily $500 \mathrm{mb}$ height fields over the Northern Hemisphere extending from the pole to $15^{\circ} \mathrm{N}$. The period under consideration is from 1 December 1972 to 1 December 1976. The values of this variable are available at each $5^{\circ}$ latitude by $10^{\circ}$ longitude grid point at 1200 GMT daily. The objective scheme that produced these grid point values from the actual station data was discussed by Kruger (1969). This data set is a portion of the ongoing collection of daily maps prepared by the Canadian Meteorological Center for daily operational forecast purposes.

A straightforward computation of the geostrophic wind field for each map was performed, viz.,

$$
u_{\theta}=\frac{-g}{2 \Omega a \sin \theta} \frac{\partial z}{\partial \theta}, \quad v_{\theta}=\frac{g}{2 \Omega a \sin \theta \cos \theta} \frac{\partial z}{\partial \lambda},
$$

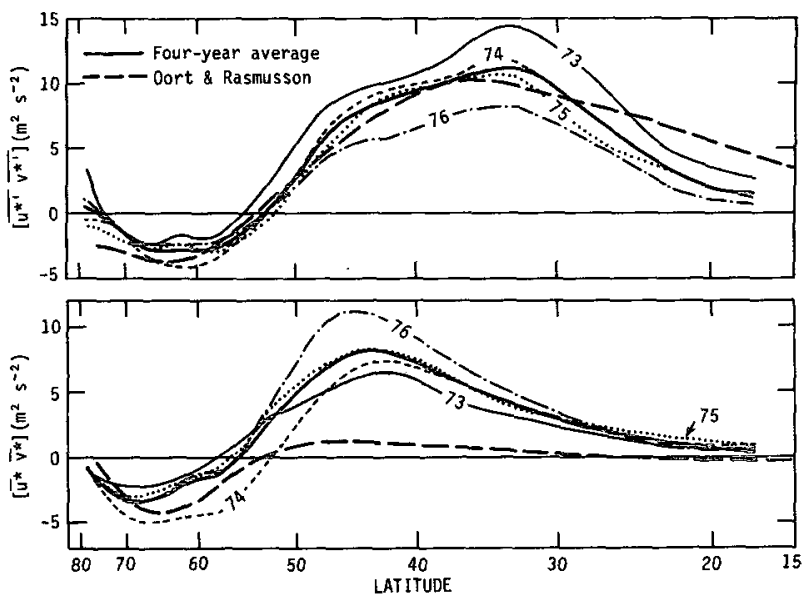

Fig. 1a, Variations with latitude of the annual or four-year average momentum flux $\left(\mathrm{m}^{2} \mathrm{~s}^{-2}\right)$ by transient eddies, $\overline{\left[u^{* / t} v^{* / 1}\right]}$, at $500 \mathrm{mb}$.

Fig. 1b. As in Fig. 1a except for the standing eddies, [ $\left[u^{*} \theta^{*}\right]$.

where $z$ is the $500 \mathrm{mb}$ height, $g$ the gravity, $\Omega$ the earth's rotation rate, $a$ the mean radius of the earth, $\theta$ the latitude and $\lambda$ the longitude. It is expected that geostrophic wind slightly overestimates the actual wind in the regions of cyclonic curvature and underestimates it in the regions of anticyclonic curvature. The overestimate for the zonal average $u$ should not be more than about $2 \mathrm{~m} \mathrm{~s}^{-1}$ at this level (OR, p. 204). From this wind field I computed the average meridional flux of zonal momentum which in general can be written as a sum of four terms:

$$
[\overline{u v}]=\left[\overline{u^{* \prime} v^{* \prime}}\right]+\left[\bar{u}^{*} \bar{v}^{*}\right]+[\bar{u}][\bar{v}]+\overline{[u]^{\prime}[\bar{v}]^{\prime}} .
$$

Standard notation is used here. A bar and a square bracket represent time and zonal averages, respectively; a prime and an asterisk represent the departures from the respective averages. The last two terms are necessarily zero for the geostrophic wind. The first two terms at middle and high latitudes are primarily due to the geostrophic part of the wind. The upper diagram of Fig. 1 shows $\left[u^{* \prime} v^{* \prime}\right]$, the momentum transport by the truly transient eddies. Values for individual years

TABLE 1. Values of $\left[\bar{u}^{*} \bar{b}^{*}\right]$ at $500 \mathrm{mb}$ in units of $\mathrm{m}^{2} \mathrm{~s}^{-2}$. NKVB's values are from their Table 4.2;

\begin{tabular}{|c|c|c|c|c|c|c|c|}
\hline $\begin{array}{l}\text { Latitude } \\
\left({ }^{\circ} \mathrm{N}\right)\end{array}$ & Dec-Feb & Mar-May & $\begin{array}{c}\text { Newell el al. } \\
\text { Jun-Aug }\end{array}$ & Sep-Nov & Average & $\begin{array}{l}\text { Oort and } \\
\text { Rasmusson }\end{array}$ & This study \\
\hline 80 & 0,6 & -0.2 & 0.1 & 0.1 & 0.15 & & \\
\hline 70 & 0.1 & -1.8 & -0.7 & -0.6 & -0.75 & -3.1 & -3.2 \\
\hline 60 & 1.4 & -2.2 & 0.0 & -1.2 & -0.50 & -3.2 & -2.0 \\
\hline 50 & 6.0 & 1.5 & 1.6 & 4.1 & 3.30 & 0.9 & 5.0 \\
\hline 40 & 8.0 & 4.9 & -0.2 & 2.8 & 3.87 & 0.9 & 7.0 \\
\hline 30 & 2.3 & 2.3 & -0.6 & 1.5 & 1.37 & 0.2 & 2.7 \\
\hline 20 & 2.3 & 0.5 & 1.7 & -0.1 & 1.10 & -0.2 & 1.0 \\
\hline
\end{tabular}
OR's from their Table C1b. 

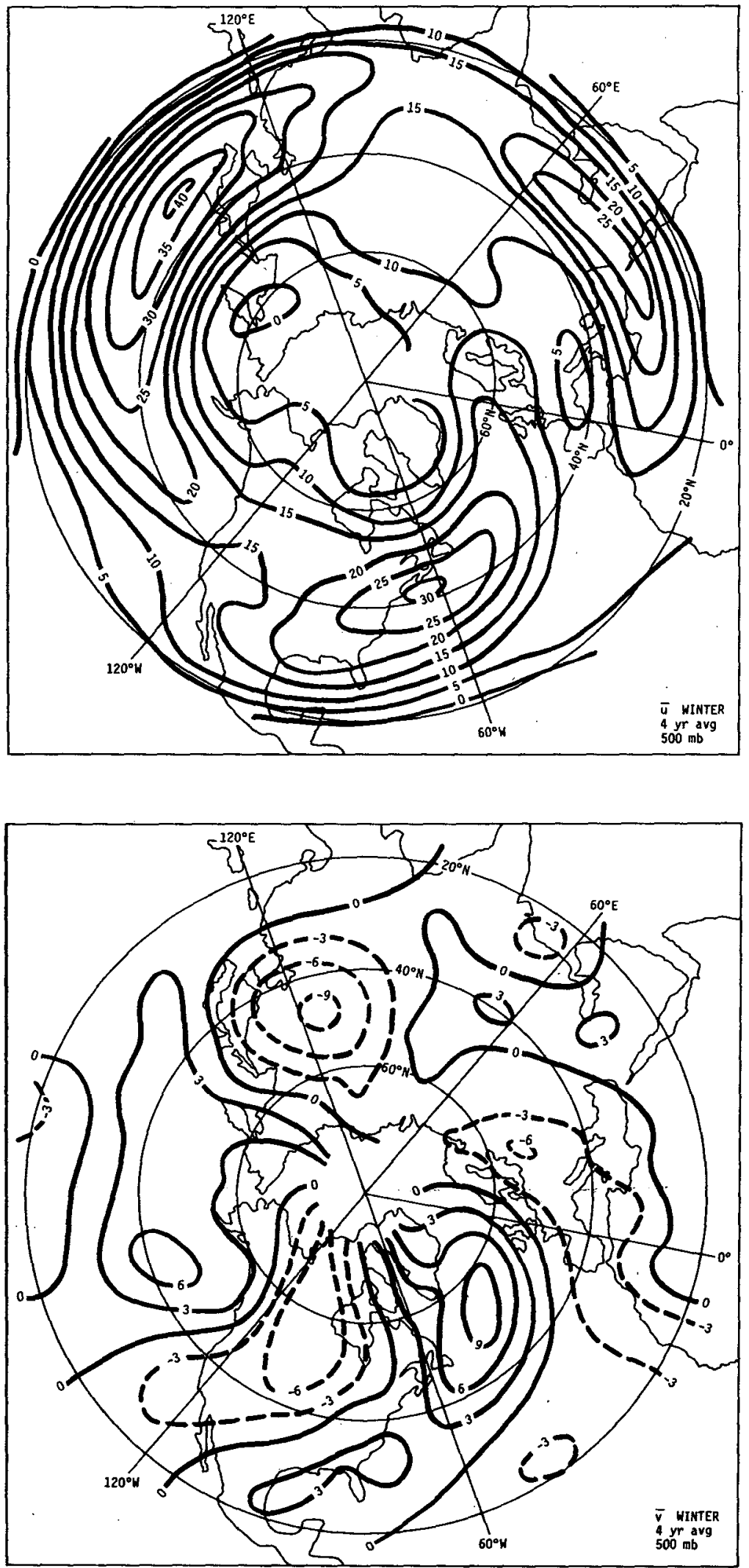

FIG. 2. The winter average zonal velocity and meridional velocity $\left(\mathrm{m} \mathrm{s}^{-1}\right)$ at $500 \mathrm{mb}$. 

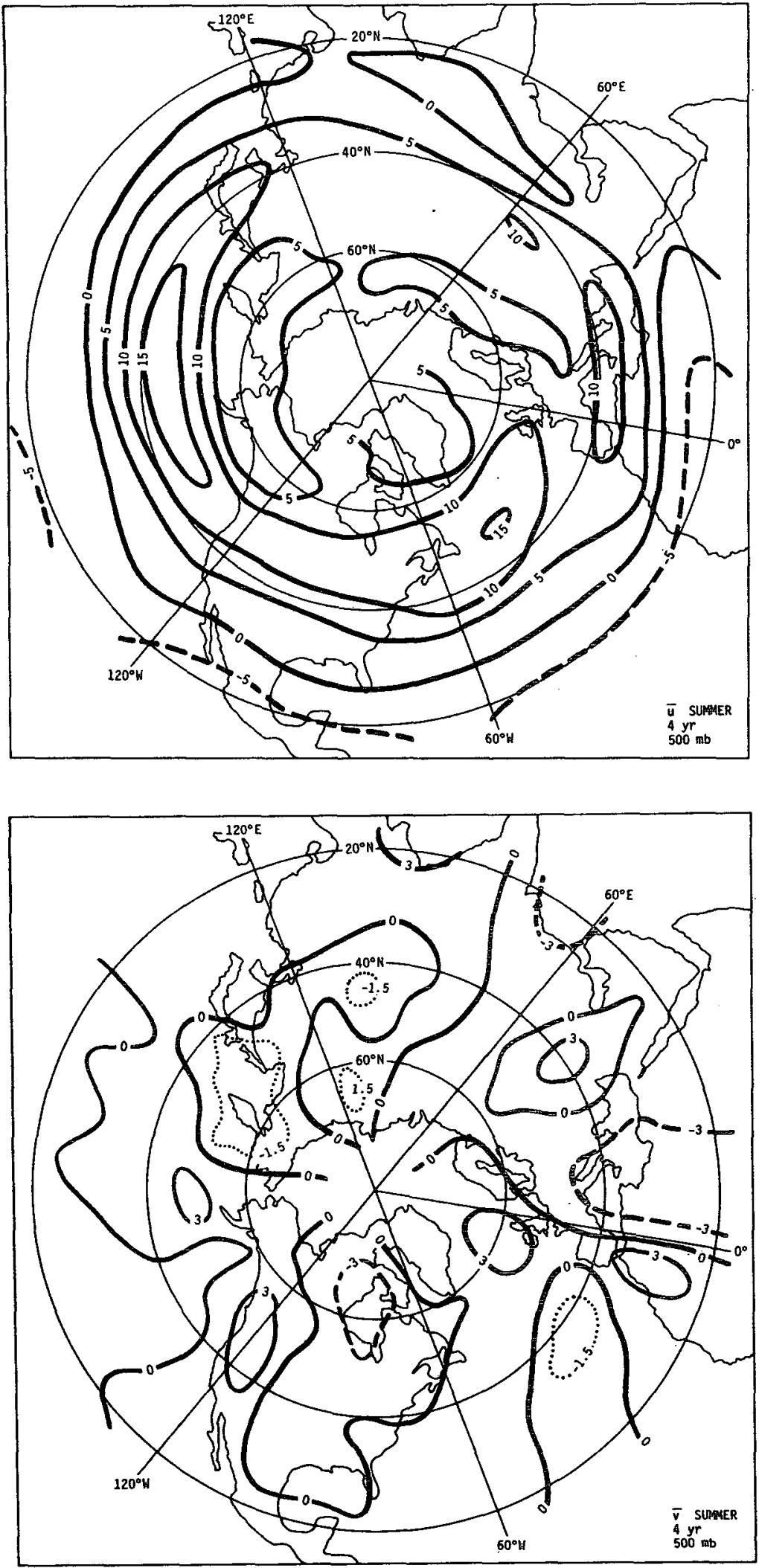

Fig. 3. As in Fig. 2 except for summer. 


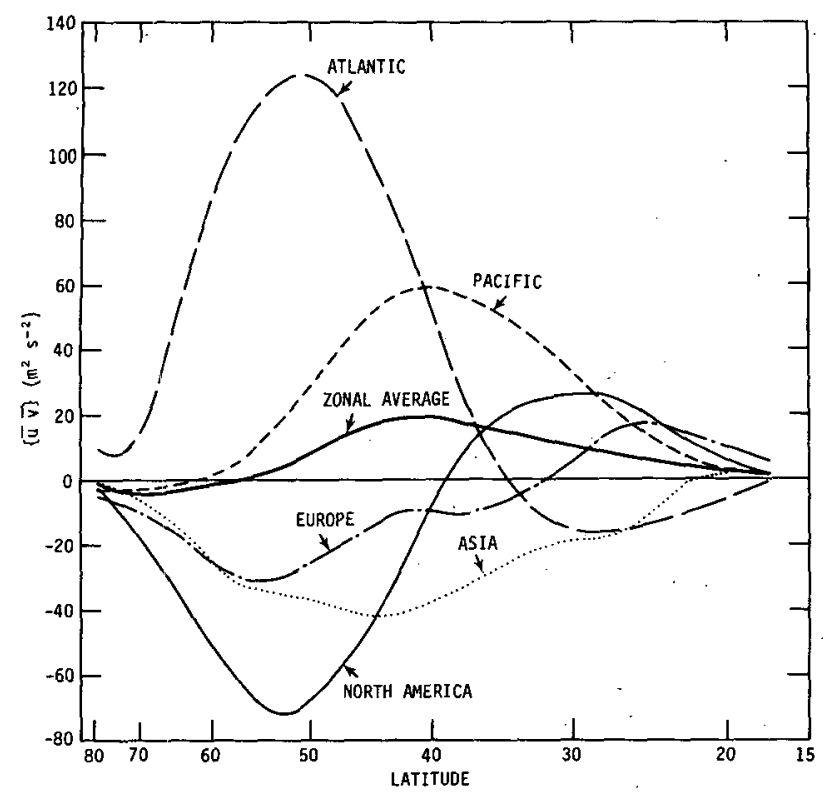

FIG. 4. Variations with latitude of the winter average $\bar{u} v$ for each of the five longitudinal sectors separated by meridions $0^{\circ}$, $60^{\circ} \mathrm{E}, 120^{\circ} \mathrm{E}, 120^{\circ} \mathrm{W}$ and $60^{\circ} \mathrm{W}$.

as well as the four-year average are given. The result for $\overline{\left[u^{\prime} v^{\prime}\right]}$ excerpted from Table C1a in OR is also plotted. Since we have $\overline{\left[u^{\prime} v^{\prime}\right]}=\overline{\left[u^{* \prime} v^{* \prime}\right]}+\overline{[u]^{\prime}[v]^{\prime}}$ and since the second term here is negligible at middle and high latitudes, one may meaningfully compare OR's curve with mine. On the other hand, the second term is expected to be significantly positive in the subtropics due to the seasonal change of the Hadley cell circulation. Fig. 1 shows good agreement between OR's values and those obtained in this study at middle and high latitudes. It also shows that OR's values are systematically larger in the subtropics as expected. Hence, the two sets of data are quite compatible as far as the transient eddies are concerned.

The lower diagram of Fig. 1 shows the corresponding values of $\left[\bar{u}^{*} \bar{v}^{*}\right]$. It clearly reveals that OR's values are very much smaller than any of my individual yearly average as well as the four-year average. The differences are particularly large between 30 and $50^{\circ} \mathrm{N}$. The values of $\left[\tilde{u}^{*} \tilde{v}^{*}\right]$ obtained by OR, NKVB and this study are summarized in Table 1. The values under "Average" should essentially reflect the yearly average values although they must slightly overestimate the latter. NKVB's or OR's values are also seen to differ significantly between one another. My values at high latitudes are more like OR's, whereas NKVB's values are in between OR's and mine at middle latitudes. These differences are too large, as well as too intriguing to be ignored. Part of the differences quite likely reflect real interannual variabilities. The results in Fig. 1 suggest that the year-to-year variability in $\overline{\left[u^{* \prime} v^{* \prime}\right]}$ is larger in the southern part of the mid-latitudes, whereas that in $\left[\bar{u}^{*} \bar{v}^{*}\right]$ is more pronounced in the northern part. Even the five-year mean values of $O R$ vary from one pentad to the next. ${ }^{1}$ Since NKVB's analysis was based on late 1950's data, OR's on mid 1960's data and mine on early 1970's data, some genuine differences among these three sets of values for $\left[\bar{u}^{*} \bar{v}^{*}\right]$ should be present. However, differences of this kind are necessarily small.

To assess the nature of the large differences among the three sets of estimates, let us find out what lies behind the large values of $\left[\tilde{u}^{*} \bar{v}^{*}\right]$ in this study. Fig. 2 shows the maps of $\bar{u}$ and $\bar{v}$ averaged over four winter seasons which are defined to be between 1 December and 28 February of the following year. The $\bar{u}$ map has all the well-known features. For instance there is the strongest westerly wind component just to the east of southern Japan; there is also a weaker maximum off the eastern Canadian coast and a still weaker maximum to the south-east of the Mediterranean Sea. These maxima probably arise from the confluence mechanism (Namias and Clapp, 1949) as elaborated by Blackmon et al. (1977). What has not been examined in sufficient detail is the role of continentality in this confluence mechanism. The $\bar{v}$ map shows a northerly component over a large portion of each continent with the exception of a region around $40^{\circ} \mathrm{N}, 60^{\circ} \mathrm{E}$ over Asia Minor. This feature is clearly a manifestation of the repeated bifurcation of the jet stream impinging upon the Himalaya-Tibetan plateau. The northerly maximum located just to the south of the southerly maximum makes this interpretation fairly convincing. The $\bar{v}$ fields over the Atlantic and Pacific oceans are decidedly southerly. The averaged summer $\bar{u}$ and $\bar{v}$ fields, defined as the average from 1 June to 31 August, are shown in Fig. 3. As expected, the flow is much weaker and the relatively strong $\bar{u}$ regions shift eastward and northward from their winter positions. The $\bar{v}$ field has finer features. But there still remains gross resemblance between the winter and summer $500 \mathrm{mb}$ flows, in spite of the reversal of surface thermal contrast between ocean and continents. Hence the contribution of $\left[\bar{u}^{*} \bar{v}^{*}\right]$ in summer does not negate that in winter.

It is the strong correlation between the southerly. flow with large $\bar{u}$ components and the northerly flow with smaller $\bar{u}$ components that gives rise to the large positive values for $\left[\bar{u}^{*} \bar{v}^{*}\right]$ at mid-latitudes as seen in Fig. 1. The standing waves clearly have planetary scales. That the quasi-steady very long waves in winter could be important has been emphasized in a number of studies (e.g., Wiin-Nielsen et al. 1963, 1964). The products of $\bar{u}$ and $\bar{v}$ at individual grid points are much larger than the zonal average value. In particular, the average of $\bar{u} \bar{v}$ over each oceanic sector has large positive values, whereas those over the continental sectors have negative values. The subdivisions according to the five meridians indicated in Fig. 2 are crudely chosen but they do bring out the systemic differences between

\footnotetext{
1 Oort (private communication).
} 
oceanic and continental regions. The actual values for each sector are shown in Fig. 4. It should be emphasized that $\bar{u}^{*} \bar{v}^{*}$ is not a very physically meaningful local property. The reason is that $\bar{u} \bar{v}$ and $\bar{u}^{*} \tilde{v}^{*}$ at the same point may and often do have opposite signs. That is why the sectorial average of $\bar{u} \bar{v}$ instead of $\bar{u}^{*} \bar{v}^{*}$ is examined. For the same reason, computational results such as that in Fig. 10a in Blackmon et al. (1977) which show the grid point values of $\bar{v}^{*} \bar{T}^{*}$ have little physical meaning at best, and might lead to misleading interpretation.

It becomes evident that $\left[\bar{u}^{*} \bar{v}^{*}\right]$ is the residue of many large positive and negative values. It therefore has great inherent uncertainty. By the nature of this uncertainty, most of it could not have been alleviated even if I had used actual wind data or the values of wind fields deduced from geopotential height data by solving the balance-equation instead of the geostrophic equation. The crucial factor that determines the reliability of $\left[\bar{u}^{*} \bar{v}^{*}\right]$ is apparently the accuracy of the relative phase between the $\bar{u}$ and $\bar{v}$ fields, rather than that of their magnitudes. It is not at all obvious that this atmospheric flow information can be truthfully retained in the data taken by the present distribution of rawinsonde stations. This is a major topic of discussion in a very recent article by Oort (1978). He found, among other things, that the large spatial data gaps due to the lack of stations over the oceans even in the Northern Hemisphere are particularly detrimental to our efforts of deducing statistics related to the stationary eddies. Such statistics tend to be grossly underestimated even though those related to the transient eddies are not nearly as sensitive to this error source. He pointed out that while the former are determined by the east-west asymmetry of the flow, the latter have a predominantly zonal character. The absence of an adequate longitudinal distribution of stations could therefore cause a considerably larger error in the former than in the latter. This problem becomes more serious if station wind data are used instead of grid data deduced from

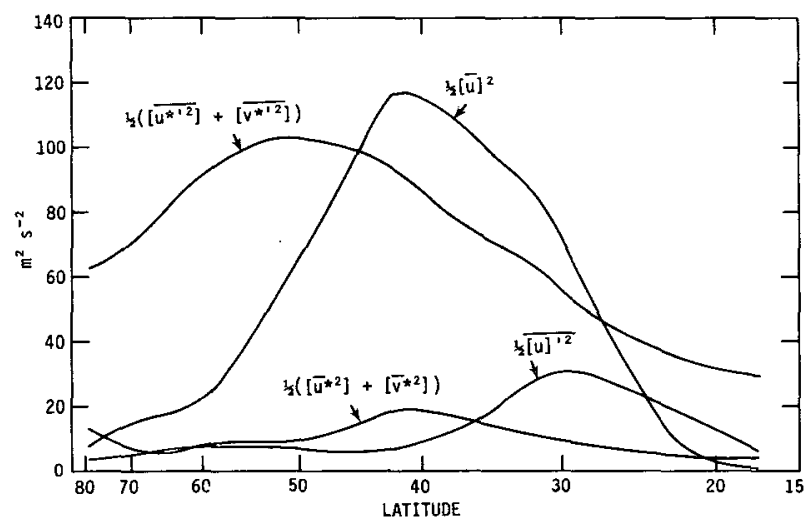

Frc. 5. The average kinetic energy $\left(\mathrm{m}^{2} \mathrm{~s}^{-2}\right)$ associated with the various components of the flow field at $500 \mathrm{mb}$. the hemispheric analyzed height field. When station data are used, the grid values are obtained by some sort of interpolation of values at stations. Hence the real maximum values like the jets would be underestimated and the real minimum values overestimated. It follows that $\left[\bar{u}^{*} \bar{v}^{*}\right]$ would be underestimated. Furthermore, if the grid values are obtained from time-averaged station data, as was done in OR and NKVB, that type of error would tend to be accumulative. This aspect of the error could be minimized if daily analyzed grid values from the height field were used as in this study.

As a concluding remark, I would say that there is no compelling reason to discount the stationary and quasisteady planetary waves as unimportant agents of transporting momentum meridionally. On the contrary, evidence shown in this study ${ }^{2}$ suggests that these waves might be important, but their effects are difficult to measure accurately. If they are truly important, it would be quite remarkable because they have only relatively little average kinetic energy as shown in Fig. 5, amounting to about $15 \%$ of that of the transient eddies.

Acknowledgments. This work was done during a part of my sabbatical leave at the Numerical Studies Division of Environment Canada, Toronto. The hospitality of the host institute and the technical assistance in using the data from a number of staff there are gratefully acknowledged. This research was supported by the Global Atmospheric Research Program, Climatic Dynamics Research Section, National Science Foundation, under Grant ATM-7401188.

\section{REFERENCES}

Blackmon, M. L., 1976: A climatological spectral study of the $500 \mathrm{mb}$ geopotential height of the Northern Hemisphere. J. Atmos. Sci., 33, 1607-1623.

- J. M. Wallace, N. C. Lau and S. L. Mullen, 1977: An observational study of the Northern Hemisphere wintertime circulation. J. Aimos. Sci., 34, 1040-1053.

Kao, S. K., and R. J. Kuczek, 1973: The kinetic energy of largescale atmospheric motion in wavenumber-frequency space: IV. The tropics. J. Atmos. Sci., 30, 308-312.

Kruger, H. B., 1969: General and special approaches to the problem of objective analysis of meteorological variables. Quart. J. Roy. Meteor. Soc., 95, 21-39.

Lorenz, E. N., 1967: The nature and theory of the general circulation of the atmosphere. WMO, $161 \mathrm{pp}$.

Namis, J., and P. F. Clapp, 1949: Confluence theory of high tropospheric jet stream. J. Meteor., 6, 330-336.

Newell, R. E., J. W. Kidson, D. G. Vincent and G. J. Boer, 1972 : The General Circulation of the Tropical Atmosphere and Interactions with Extratropical Latitudes, Vol. I. The MIT Press, $258 \mathrm{pp}$.

\footnotetext{
${ }^{2}$ It was brought to my attention by one reviewer that NgarCheung Lau at the University of Washington has just completed, among other things, similar computations using 10 years of NMC grid data. His $\left[\tilde{u}^{*} \tilde{0}^{*}\right]$ values are slightly more than $50 \%$ of the $\overline{\left[u^{\prime} v^{\prime}\right]}$ values at all levels. His winter $\bar{u}$ and $\delta$ maps are quantitatively similar to my Figs. $2 a$ and $2 b$.
} 
Oort, A. H., 1978: Accuracy of the rawinsonde network for global circulation studies tested through numerical model output. Mon. Wea. Rev., 106, (in press).

- , and E. M. Rasmusson, 1971: Atmospheric circulation statistics. NOAA Prof. Pap. 5, U. S. Dept. of Commerce, 323 pp.

Pratt, R. W., 1977: Space-time kinetic energy spectra in midlatitudes. $J$. Atmos. Sci., 34, 1054-1057.
- , and J. M. Wallace, 1976: Zonal propagation characteristics of large-scale fluctuations in the mid-latitude troposphere. J. Atmos. Sci., 33, 1184-1194.

Wiin-Nielsen, A., J. A. Brown and M. Drake, 1963 : On the atmospheric energy conversions between the zonal flow and the eddies. Tellus, 15, 261-279.

,-- and,- 1964: Further studies of energy exchange between the zonal flow and the eddies. Tellus, 16, 168-180. 